\title{
Chiral Ward identities and pion propagation at finite temperature in the linear sigma model
}

\author{
Alejandro Ayala* \\ Instituto de Ciencias Nucleares, Universidad Nacional Autónoma de México, \\ Apartado Postal 70-543, México Distrito Federal 04510, México. \\ E-mail: ayala@nuclecu.unam.mx
}

ABSTRACT: Working within the linear sigma model at finite temperature, we construct effective one-loop vertices and propagators satisfying the chiral Ward identities, for small pion momentum and mass compared to the sigma mass. We obtain an expansion for the pion dispersion relation up to second order in the parameter $m_{\pi}^{2} / 4 \pi^{2} f_{\pi}^{2}$. At leading order, this expansion reproduces the dispersion curve obtained in chiral perturbation theory. We also study pion damping and compute the mean free path for pions traveling in the medium before forming a sigma resonance. These results are also in good agreement with chiral perturbation theory when using a value for the sigma mass of $600 \mathrm{MeV}$.

\section{KEYwORDS: 'chiral symmetries; pion production; finite temperature field theory'}

\section{Introduction}

In recent years, a considerable effort has been devoted to the production and detection of the so called quark-gluon plasma -the state of matter where the basic QCD degrees of freedom are not confined to a single hadron- in relativistic heavyion collisions. The disentanglement of the signals to discern between different outcomes of the collision, requires the understanding of the evolution of the hadronic state from production to freeze out. Since pions are the most copiously produced particles in this kind of collisions, a proper understanding of their propagation properties within a dense a hot environment represents a key ingredient for the understanding of the system's physical properties as a whole.

In general, the hadronic degrees of freedom are accounted for by means of effective chiral theories whose basic ingredient is the fact that pions are the Goldston bosons coming from the spontaneous breakdown of chiral symmetry. Chiral perturbation theory (ChPT) is one of such theories that has been employed to show a number of properties exhibited by pions at finite tempera-

\footnotetext{
${ }^{*}$ In collaboration with S. Sahu and M. Napsuciale
}

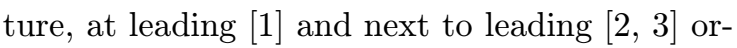
ders. A striking result obtained from the second order calculations is that the shift in the temperature dependence of the pion mass is opposite in sign and about three times larger in magnitude than the first order shift, already at temperatures of order of $150 \mathrm{MeV}$. This result might signal either the breakdown of the perturbative scheme at such temperatures or the need to compute beyond next to leading order in ChPT, given the large relative corrections found.

Nevertheless, the simplest realization of chiral symmetry is still provided by the much studied linear sigma model [4] able field theory at zero [inj and (consequently) at finite temperature $[\overline{6}|\overline{1}|$. The model has been the subject of a renewed interest in view of recent theoretical results $\overline{\overline{1}} \overline{1}$ and analyses of data that seem to confirm the existence of a broad scalar resonance, with a mass in the vicinity of $600 \mathrm{MeV}$, that can be identified with the $\sigma$-meson. If confirmed, this last feature could place the linear sigma model above the status of a toy model to study QCD below the deconfinement scale. It thus becomes important to check that the results obtained by means of other theories 
such as ChPT can be reproduced by the linear sigma model when working in a given kinematical regime.

Here we show that the linear sigma model reproduces the leading order modification to the pion mass in a thermal pion medium obtained in ChPT at leading order, when use is made of a systematic expansion in the ratio $m_{\pi}^{2} / m_{\sigma}^{2}$ at zeroth order -with $m_{\sigma}, m_{\pi}$ the vacuum sigma and pion masses, respectively-and when treating the momentum and temperature as small quantities of order $m_{\pi}$. In this regime, the effective expansion parameter becomes $m_{\pi}^{2} / 4 \pi^{2} f_{\pi}^{2}$, with $f_{\pi}$ the pion decay constant. Such parameter allows for a controlled loop expansion of the pion self-energy from where we can study both, the modification to the pion mass at next to leading order and pion damping in the elastic and inelastic channels. This is accomplished by constructing one-loop effective vertices and propagators satisfying the chiral Ward identities and thus respecting chiral symmetry. Details of the calculation as well as a more extended discussion on its implications can be found in Refs.

\section{Effective vertices and chiral Ward identities}

The Lagrangian for the linear sigma model, including only the meson degrees of freedom and after the explicit inclusion of the chiral symmetry breaking term, can be written as [i,

$$
\begin{aligned}
\mathcal{L} & =\frac{1}{2}\left[(\partial \pi)^{2}+(\partial \sigma)^{2}-m_{\pi}^{2} \pi^{2}-m_{\sigma}^{2} \sigma^{2}\right] \\
& -\lambda^{2} f_{\pi} \sigma\left(\sigma^{2}+\pi^{2}\right)-\frac{\lambda^{2}}{4}\left(\sigma^{2}+\pi^{2}\right)^{2}
\end{aligned}
$$

where $\pi$ and $\sigma$ are the pion and sigma fields, respectively, and the coupling $\lambda^{2}$ is given by

$$
\lambda^{2}=\frac{m_{\sigma}^{2}-m_{\pi}^{2}}{2 f_{\pi}^{2}}
$$

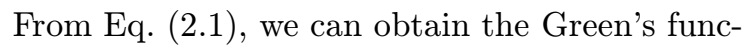
tions and Feynman rules to use in perturbative calculations. In particular, the bare pion and sigma propagators $\Delta_{\pi}(P), \Delta_{\sigma}(Q)$ and the bare one-sigma two-pion and four-pion vertices $\Gamma_{12}^{i j}$, $\Gamma_{04}^{i j k l}$ are given by (hereafter, capital Roman let- ters are used to denote four momenta)

$$
\begin{aligned}
i \Delta_{\pi}(P) \delta^{i j} & =\frac{i}{P^{2}-m_{\pi}^{2}} \delta^{i j} \\
i \Delta_{\sigma}(Q) & =\frac{i}{Q^{2}-m_{\sigma}^{2}} \\
i \Gamma_{12}^{i j} & =-2 i \lambda^{2} f_{\pi} \delta^{i j} \\
i \Gamma_{04}^{i j k l} & =-2 i \lambda^{2}\left(\delta^{i j} \delta^{k l}\right. \\
& \left.+\delta^{i k} \delta^{j l}+\delta^{i l} \delta^{j k}\right) .
\end{aligned}
$$

These are sufficient to obtain the modification to the pion propagator, both at zero and finite temperature, at any given perturbative order.

Alternatively, we can exploit the relations that chiral symmetry imposes among different $\mathrm{n}$ point Green's functions. These relations, known as chiral Ward identities (ChWIs), are a direct consequence of the fact that the divergence of the axial current may be used as an interpolating field for the pion [ivin. Thus, one could construct the modification to one of the above Green's functions at a given perturbative order and from there, build up the induced modification to other Green's functions related to the former by a ChWI. For example, two of the ChWIs satisfied -order by order in perturbation theoryby the functions $\Delta_{\pi}(P), \Delta_{\sigma}(Q), \Gamma_{12}^{i j}$ and $\Gamma_{04}^{i j k l}$ are

$$
\begin{aligned}
f_{\pi} \Gamma_{04}^{i j k l}\left(; 0, P_{1}, P_{2}, P_{3}\right) & =\Gamma_{12}^{k l}\left(P_{1} ; P_{2}, P_{3}\right) \delta^{i j} \\
& +\Gamma_{12}^{l j}\left(P_{2} ; P_{3}, P_{1}\right) \delta^{i k} \\
& +\Gamma_{12}^{j k}\left(P_{3} ; P_{1}, P_{2}\right) \delta^{i l} \\
\Gamma_{12}^{i j}(Q ; 0, P)=f_{\pi}^{-1}\left[\Delta_{\sigma}^{-1}(Q)\right. & \left.-\Delta_{\pi}^{-1}(P)\right] \delta^{i j},
\end{aligned}
$$

where momentum conservation at the vertices is implied, that is $P_{1}+P_{2}+P_{3}=0$ and $Q+P=0$.

At one loop and after renormalization, we recall that the sigma propagator is modified by finite terms. At zero temperature this modification is purely imaginary and its physical origin is that a sigma particle, with a mass larger than twice the mass of the pion, is unstable and has a (large) non-vanishing width coming from its decay channel into two pions. At finite temperature the modification results in real and imaginary parts. The real part modifies the sigma dispersion curve whereas the imaginary part represents a temperature dependent contribution to the sigma width. 
In an expansion in the parameter $m_{\pi}^{2} / m_{\sigma}^{2}$, the leading contribution to the sigma self-energy comes from the sigma polarization bubble where the intermediate particles are pions. Working in the imaginary-time formalism of Thermal Field Theory, this is given as $6 \lambda^{4} f_{\pi}^{2} I(\omega, q)$ where the function $I$ is defined by

$$
\begin{aligned}
I(\omega, q)= & T \sum_{n} \int \frac{d^{3} k}{(2 \pi)^{3}} \frac{1}{\omega_{n}^{2}+k^{2}+m_{\pi}^{2}} \\
& \frac{1}{\left(\omega_{n}-\omega\right)^{2}+(\mathbf{k}-\mathbf{q})^{2}+m_{\pi}^{2}} .
\end{aligned}
$$

Here $\omega=2 m \pi T$ and $\omega_{n}=2 n \pi T$ ( $m, n$ integers) are discrete boson frequencies and $q=|\mathbf{q}|$. From Eq. $(2.5)$ we obtain the time-ordered version $I^{t}$ of the function $I$, after analytical continuation to Minkowski space. The imaginary part of $I^{t}$ is given by

$$
\begin{aligned}
\operatorname{Im} I^{t}\left(q_{0}, q\right) & =\frac{\varepsilon\left(q_{o}\right)}{2 i}\left[I\left(i \omega \rightarrow q_{o}+i \epsilon, q\right)\right. \\
& \left.-I\left(i \omega \rightarrow q_{o}-i \epsilon, q\right)\right] \\
& =-\frac{1}{16 \pi}\left\{a\left(Q^{2}\right)\right. \\
& \left.+\frac{2 T}{q} \ln \left(\frac{1-e^{-\omega_{+}\left(q_{o}, q\right) / T}}{1-e^{-\omega_{-}\left(q_{0}, q\right) / T}}\right)\right\} \\
& \times \Theta\left(Q^{2}-4 m_{\pi}^{2}\right),
\end{aligned}
$$

where $Q^{2}=q_{0}^{2}-q^{2}, \varepsilon$ and $\Theta$ are the sign and step functions, respectively, and the functions $a$ and $\omega_{ \pm}$are given by

$$
\begin{aligned}
a\left(Q^{2}\right) & =\sqrt{1-\frac{4 m_{\pi}^{2}}{Q^{2}}} \\
\omega_{ \pm}\left(q_{0}, q\right) & =\frac{\left|q_{0}\right| \pm a\left(Q^{2}\right) q}{2},
\end{aligned}
$$

whereas the real part of $I^{t}$ at $Q=0$ is given by

$$
\operatorname{Re} I^{t}(0)=-\frac{1}{8 \pi^{2}} \int_{0}^{\infty} \frac{d k}{E_{k}}\left[1+2 f\left(E_{k}\right)\right],
$$

where $E_{k}=\sqrt{k^{2}+m_{\pi}^{2}}$ and the function $f$ is the Bose-Einstein distribution

$$
f\left(E_{k}\right)=\frac{1}{e^{E_{k} / T}-1} .
$$

Therefore, the one-loop effective sigma propagator becomes

$$
i \Delta_{\sigma}^{\star}(Q)=\frac{i}{Q^{2}-m_{\sigma}^{2}+6 \lambda^{4} f_{\pi}^{2} I^{t}(Q)} .
$$

The temperature-independent infinities are absorbed into the redefinition of the physical masses and coupling constants by the introduction of suitable counterterms in the usual manner.

In order to preserve the ChWIs expressed in Eq. (2. 2.4 , the corresponding one-loop effective one-sigma two-pion and four-pion vertices are

$$
\begin{array}{cl}
i \Gamma_{12}^{\star i j}\left(Q ; P_{1}, P_{2}\right)= & -2 i \lambda^{2} f_{\pi} \delta^{i j} \\
& {\left[1-3 \lambda^{2} I^{t}(Q)\right],} \\
i \Gamma_{04}^{\star i j k l}\left(; P_{1}, P_{2}, P_{3}, P_{4}\right)= & -2 i \lambda^{2} \times \\
\{[1-3 & \left.\lambda^{2} I^{t}\left(P_{1}+P_{2}\right)\right] \delta^{i j} \delta^{k l} \\
+[1-3 & \left.\lambda^{2} I^{t}\left(P_{1}+P_{3}\right)\right] \delta^{i k} \delta^{j l} \\
+[1-3 & \left.\left.\lambda^{2} I^{t}\left(P_{1}+P_{4}\right)\right] \delta^{i l} \delta^{j k}\right\} .
\end{array}
$$

The functions in Eq. (i2. $\left.1 \overline{1} \mathbf{1}_{-1}\right)$ arise from considering all of the possible one-loop contributions to the one-sigma two-pion and four-pion vertices, when maintaining only the zeroth order terms in a systematic expansion in the parameter $m_{\pi}^{2} / m_{\sigma}^{2}$.

\section{Dispersion relation}

We now use the above effective vertices and propagator to construct the one-loop modification to the pion self-energy. Keeping only the leading order contribution when considering $m_{\pi}, T$ and $P$ as small compared to $m_{\sigma}$ and to zeroth order in $m_{\pi}^{2} / m_{\sigma}^{2}$ for which

$$
\lambda^{2}\left(1-\frac{2 \lambda^{2} f_{\pi}^{2}}{m_{\sigma}^{2}}\right) \approx \frac{m_{\pi}^{2}}{2 f_{\pi}^{2}},
$$

the pion self-energy can be written as

$$
\begin{aligned}
\Pi(P) & =\left(\frac{m_{\pi}^{2}}{2 f_{\pi}^{2}}\right) T \sum_{n} \int \frac{d^{3} k}{(2 \pi)^{3}} \frac{1}{K^{2}+m_{\pi}^{2}} \\
& \left\{5+2\left(\frac{P^{2}+K^{2}}{m_{\pi}^{2}}\right)\right. \\
& \left.-\left(\frac{m_{\pi}^{2}}{2 f_{\pi}^{2}}\right)\left[9 I^{t}(0)+6 I^{t}(P+K)\right]\right\},
\end{aligned}
$$

with $K=\left(\omega_{n}, \mathbf{k}\right)$ and $P=(\omega, \mathbf{p})$. The pion dispersion relation is thus obtained from the solution to

$$
P^{2}+m_{\pi}^{2}+\operatorname{Re} \Pi(P)=0
$$


after the analytical continuation $i \omega \rightarrow p_{0}+i \epsilon$. The temperature dependent infinities contained in Eq. (3.2-i) are exactly canceled by the contribution from the terms necessary to introduce at one loop to carry the (vacuum) renormalization procedure $\overline{9}$.

Let us first look at the dispersion relation at leading order, this results from

$$
p_{0}^{2}=p^{2}+m_{\pi}^{2}\left[1+\xi g\left(T / m_{\pi}\right)\right],
$$

where $\xi=m_{\pi}^{2} / 4 \pi^{2} f_{\pi}^{2} \ll 1$ and the function $g$ is given by

$$
g\left(T / m_{\pi}\right)=\int_{0}^{\infty} \frac{d x x^{2}}{\sqrt{1+x^{2}}} f\left(m_{\pi} \sqrt{1+x^{2}}\right) .
$$

This coincides with the result obtained in Ref. [1, ]1] by means of ChPT.

We now look at the next to leading order terms in Eq. (3.2). The first of these is purely real and represents a constant, second order shift to the pion mass squared. The remaining second order term shows a non-trivial dependence on $P$. It involves the function $S$ defined by

$$
S(P) \equiv T \sum_{n} \int \frac{d^{3} k}{(2 \pi)^{3}} \frac{I^{t}(P+K)}{K^{2}+m_{\pi}^{2}} .
$$

The integration in Eq. (3.6) can only be performed numerically. Including all the terms, the dispersion relation up to next to leading order, for $T \sim m_{\pi}$ and in the small momentum region is obtained as the solution to

$$
\begin{aligned}
p_{0}^{2} & =p^{2}+\left\{1+\xi g\left(T / m_{\pi}\right)+\frac{\xi^{2}}{2} g\left(T / m_{\pi}\right)\right. \\
& \left.\times\left[9 h\left(T / m_{\pi}\right)-4 g\left(T / m_{\pi}\right)\right]\right\} m_{\pi}^{2} \\
& +\xi^{2} \tilde{S}\left(p_{0}, p\right) .
\end{aligned}
$$

where the function $h$ is given by

$$
h\left(T / m_{\pi}\right) \equiv \int_{0}^{\infty} \frac{d k}{E_{k}} f\left(E_{k}\right) .
$$

and $\tilde{S}\left(p_{0}, p\right)=-\left(24 \pi^{4}\right) \operatorname{Re} S^{r}\left(p_{0}, p\right)$. Figure 1 shows the temperature dependence of $m_{\pi}$ obtained as the solution to Eq. (3.7i) in the limit when $p$ goes to zero. Notice that the second order correction in the parameter $\xi$ has the same sign as the first order correction. This result is opposite to the temperature behavior of the pion mass found in Refs. [inin.

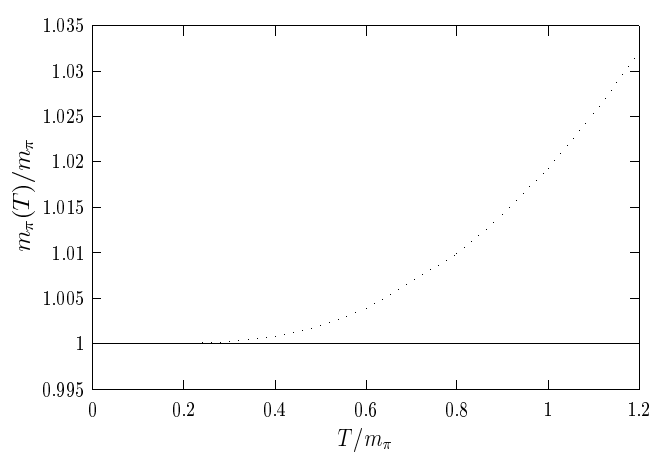

Figure 1: Temperature dependence of $m_{\pi}$ to second order in the parameter $\xi$. For comparison, the vacuum value is shown as a horizontal line.

\section{Damping}

Damping is due to two possible kinds of processes: formation of resonances and pion elastic scattering. At temperatures on the order of the pion mass, pion elastic scattering is subdominant since scattering among pions nearly disappears as the relative momentum approaches zero. In the linear sigma model, this feature is explicitly realized, for the kinematical regime discussed here, by the description of pion elastic scattering as a second order process in the small parameter $\xi$. We start by first looking at resonance formation. This process is the inverse of the one describing sigma decay (except that the former does not happen at $\mathrm{T}=0$ ). The relevant diagram to compute resonance formation is the sunset pion self energy with an intermediate sigma line and its explicit expression is

$$
\Pi_{1}(P)=4 \lambda^{4} f_{\pi}^{2} I\left(P ; m_{\pi}, m_{\sigma}\right),
$$

where $I$ is the function defined in Eq. (2. $(2 . \overline{5})$ that we rewrite here in terms of the quantities $E_{\pi}=$ $\sqrt{k^{2}+m_{\pi}^{2}}$ and $E_{\sigma}=\sqrt{(\mathbf{k}-\mathbf{p})^{2}+m_{\sigma}^{2}}$ as

$$
\begin{aligned}
I\left(P ; m_{\pi}, m_{\sigma}\right)= & T \sum_{n} \int \frac{d^{3} k}{(2 \pi)^{3}} \frac{1}{\omega_{n}^{2}+E_{\pi}^{2}} \\
& \frac{1}{\left(\omega_{n}-\omega\right)^{2}+E_{\sigma}^{2}} .
\end{aligned}
$$

The decay rate $\Gamma_{1}^{>}$is given in terms of $\operatorname{Im} \Pi_{1}$ by

$$
\Gamma_{1}^{>}(p)=-\frac{e^{p_{0} / T}}{\left(e^{p_{0} / T}-1\right)} \frac{\operatorname{Im} \Pi_{1}\left(p_{0}, p\right)}{p_{0}} .
$$


where $p_{0}=\sqrt{p^{2}+m_{\pi}^{2}}$. Im $\Pi_{1}$ contains both creation and decay rates and the factor

$$
e^{p_{0} / T} /\left(e^{p_{0} / T}-1\right)
$$

in Eq. (4.3) eliminates the piece describing pion creation. We can now compute the mean free path $\lambda$ for a pion traveling in the medium before forming a sigma resonance. This is given in terms of $\Gamma_{1}^{>}$by

$$
\lambda=\frac{v}{\Gamma_{1}^{>}(p)}=-\frac{\left(e^{p_{0} / T}-1\right)}{e^{p_{0} / T}} \frac{p}{\operatorname{Im} \Pi_{1}\left(p_{0}, p\right)}
$$

where $v=p / p_{0}$ is the magnitude of the pion's velocity. Figure 2 shows plots of $\lambda$ for three different temperatures and a value of $m_{\sigma}=600$ $\mathrm{MeV}$ as a function of the pion momentum. $\lambda$ reaches a maximum for $p \sim 0.4 m_{\pi}$. Notice that the position of the maximum is approximately independent of $T$. Except for the location of the maximum, the behavior of the curves is both in qualitative and quantitative agreement with the corresponding result obtained in ChPT [i]

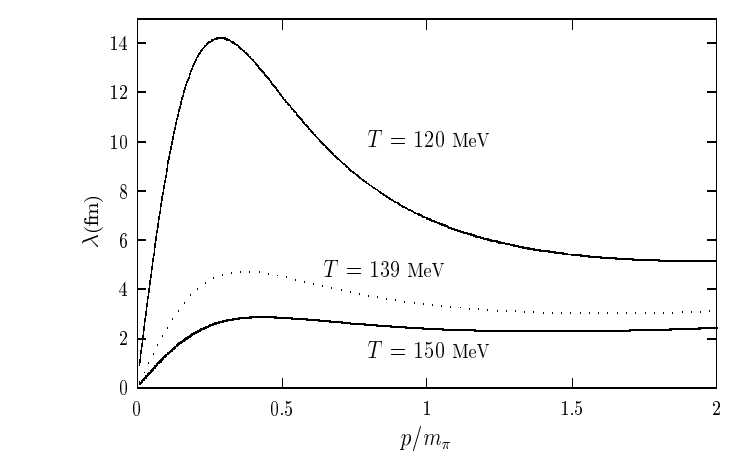

Figure 2: Mean free path as a function of momentum for a pion to travel the medium before forming

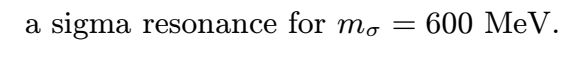

The pion elastic scattering rate is given in terms of the imaginary part of the two-loop pion self-energy $\Pi$ given in Eq. (13.2i)

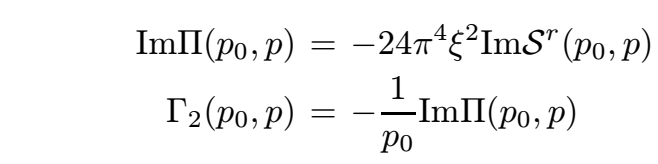

\section{Conclusions}

In conclusion, working in the linear sigma model at finite temperature, we have found effective one-loop sigma propagator and one-sigma twopion and four-pion vertices satisfying the ChWIs when maintaining only the zeroth order terms in an expansion in the parameter $m_{\pi}^{2} / m_{\sigma}^{2}$. We have used these objects to compute the two-loop order correction to the pion propagator in a pion medium for small momentum and for $T \sim m_{\pi}$ and showed that the linear sigma model yields the same result as ChPT at leading order in the parameter $\xi=m_{\pi}^{2} / 4 \pi^{2} f_{\pi}^{2}$. We have shown that, contrary to the result in Refs. order correction to the pion mass is proportional to $\xi^{2}$ and that this correction is of the same sign as the one-loop correction. The shape of the dispersion curve is not significantly altered in the kinematical regime considered. The results for pion damping are also in quantitative agreement with ChPT when using a value for $m_{\sigma}=600$ $\mathrm{MeV}$.

\section{Acknowledgments}

Partial support for this work has been received by CONACyT México under grant number 32279-E.

\section{References}

[1] J. Gasser and H. Leutwyler, 'Phys. Lett. B $\mathbf{1 8 4}$ $(1987) 83_{1 ;}$ J. L. Goity and H. Leutwyler, IPhys. Lett. B $228(1989) 517$

[2] A. Schenk, 'Phys. Rev. D 47 (1993) 5138

[3] D. Toublan, 'Phys. Rev. D $\mathbf{5 6}(1997) 5629$ r

[4] J. Schwinger, Ann. Phys. (NY) $\mathbf{2}(1958) 407$. Gell-Mann and M. Lévy, 'Nuovo Cim. 16 (1960)' $70 \overline{5}$

[5] B. W. Lee, "Chiral Dynamics" (Gordon and Breach, 1972).

[6] L. R. R. Mohan, Phys. Rev. D 14 1976$) 2670$.

[7] N. A. Tornqvist and M. Roos, iPhys. Rev.1 Lett. $\mathbf{7 6}(1996)-1575 ; \quad$ N. A. Tornqvist hep-ph/0008135.

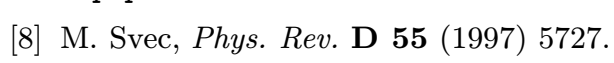

[9] A. Ayala, S. Sahu and M. Napsuciale, iPhys.' - and as-anticipated, this rate turns out to be about- - _ - tw that it is proportional to $\xi^{2}[\overline{9} \mid]$. 